\title{
Interventional bronchoscopic therapy in adult patients with tracheobronchial schwannoma
}

\author{
Beibei Jin, Ting Wang, Juan Wang, Xiaojian Qiu, Yinghua Pei, Yuling Wang, Jie Zhang \\ Department of Pulmonary and Critical Care Medicine, Beijing Tiantan Hospital, Capital Medical University, Beijing, China \\ Contributions: (I) Conception and design: All authors; (II) Administrative support: J Zhang, X Qiu; (III) Provision of study materials or patients: B \\ Jin, T Wang, J Wang, Y Pei, Y Wang; (IV) Collection and assembly of data: B Jin, T Wang; (V) Data analysis and interpretation: B Jin, T Wang, J \\ Zhang; (VI) Manuscript writing: All authors; (VII) Final approval of manuscript: All authors. \\ Correspondence to: Ting Wang, MD. Department of Pulmonary and Critical Care Medicine, Beijing Tiantan Hospital, Capital Medical University, \\ No. 119, South Fourth Ring Road West, Fengtai District, Beijing 100070, China. Email: wangting@bjtth.org.
}

\begin{abstract}
Backgrounda Tracheobronchial schwannomas are extremely rare tumors of neurogenic origin. Treatment includes surgery and interventional bronchoscopic therapy. Studies that have described interventional bronchoscopic therapy for tracheobronchial schwannoma have been reported in the published literature, but most of them are individual case reports, in which the long-term efficacy and recurrence are poorly understood. This study aimed to explore the feasibility, efficacy, and safety of interventional bronchoscopic therapy in adult patients with tracheobronchial schwannoma.
\end{abstract}

Methods: Patients with pathologically diagnosed tracheobronchial schwannoma between January 2007 and December 2020 who underwent interventional bronchoscopic therapy in a single center in China were retrospectively reviewed. The clinical features, interventional bronchoscopic therapy procedures, complications, and follow-up results were analyzed.

Results: A total of 7 patients (5 men and 2 women; median age, 56 years; age range, 26-63 years) were enrolled. Patients' symptoms were all significantly relieved after interventional bronchoscopic therapy, with

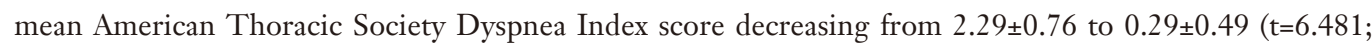
$\mathrm{P}=0.001)$. No severe procedure-related complications were observed. Intraluminal tumors were found subsequently in three cases during a short-term follow-up (4 days to 1 month). One patient underwent surgery; thereafter got lost to follow-up, while the other 2 patients underwent a second endoscopic resection with good prognosis. Six patients received long-term follow-up (range, 2-12 years; median period, 6 years), with all patients being in stable conditions.

Conclusions: Interventional bronchoscopic therapy is an acceptable approach in the management of tracheobronchial schwannoma, and is a useful alternative to surgical resection, especially for those who are at a high risk of surgery or unwilling to undergo surgery. However, long-range bronchoscopic surveillance is necessary because of the possibility of tumor recurrence. Due to its benign nature, repeat endoscopic resection can still be considered after recurrence.

Keywords: Trachea; bronchus; schwannoma; bronchoscopy; treatment

Submitted Mar 18, 2021. Accepted for publication May 10, 2021.

doi: 10.21037/apm-21-630

View this article at: http://dx.doi.org/10.21037/apm-21-630

\section{Introduction}

Tracheobronchial schwannomas are extremely rare tumors of neurogenic origin (1). Clinical and radiological presentations are non-specific (2). The rarity and non- specific symptoms of these benign primary tracheal tumors lead to an undefined optimal treatment (3). Surgery (sleeve excision or tracheal resection) or interventional bronchoscopic therapy is all commonly accepted 
treatment modalities (2). However, surgical resection and reconstruction cannot be performed sometimes because of poor patient condition. Interventional bronchoscopic therapy (such as laser, electrocautery, snaring, argon plasma coagulation, cryotherapy, etc.) is another option with less injury.

Studies that described interventional bronchoscopic therapy for tracheobronchial schwannoma have been reported in the published literature, but most of them were individual case reports (4-7), in which the long-term efficacy and recurrence are poorly understood. Hence, we aimed to better address these limitations by retrospectively reviewing tracheobronchial schwannoma patients who underwent interventional bronchoscopic therapy in a single center in China over a period of 12 years. Based on the literature reports and our clinical experience, we sought to explore the feasibility, efficacy, and safety of interventional bronchoscopic therapy for tracheobronchial schwannoma.

We present the following article in accordance with the STROBE reporting checklist (available at http://dx.doi. org/10.21037/apm-21-630).

\section{Methods}

The study was approved by the Institutional Review Board of Beijing Tian Tan Hospital (approval ID: JS2013-00702). All patients provided written informed consent for the data to be used in this study. The study was conducted in accordance with the Declaration of Helsinki (as revised in 2013).

\section{Patients}

All patients with pathologically diagnosed benign tracheobronchial schwannoma between January 2007 and December 2018, who underwent interventional bronchoscopic therapy at bronchoscopy center of Beijing Tiantan Hospital, Capital Medical University were retrospectively reviewed.

Histochemically, the resected specimen tumor postinterventional had features consistent with benign schwannoma (8): exhibited an Antoni A growth pattern (composed of spindle cells with elongated palisading nuclei); a few hyalinized blood vessels, whereas no clear necrosis or mitotic activity was observed within these areas; positive for $\mathrm{S} 100$ protein expression diffusely, but was negative for cytokeratin, desmin, cluster of differentiation 117 and smooth muscle actin.
Data including demographics, clinical presentation, tumor locations, radiologic and bronchoscopic findings, interventional bronchoscopic modalities, technical success, and complications, were obtained from electronic medical records and bronchoscopy records.

\section{The endoscopic excision procedure}

All endoscopic excision procedures were performed under general anesthesia in the operating room. Noninvasive blood pressure, pulse oximetry, and dynamic electrocardiography were monitored throughout the treatment process. Laryngeal mask (cases 1, 6, and 7), endotracheal tube (cases 2 and 4), or rigid bronchoscopy (cases 3 and 5) were used as endoscopic approaches, depending on the size and location of the tumor. An anesthesia machine or high-frequency jet ventilator was used for ventilation, and pulse oxygen saturation was monitored and maintained above $95 \%$ during the operation.

Interventional bronchoscopic therapy was performed using an electronic bronchoscope (BF-1T260; Olympus, Tokyo, Japan). Pedunculated schwannoma was usually excised using an electrocautery snare (ERBE, Tubingen, Germany), or as several pieces, and sessile schwannomas were usually excised along their base using a high-frequency electric knife (ERBE, Tubingen, Germany). The excised tissues were grasped using biopsy forceps or cryoprobe and removed using a rigid bronchoscope (Karl Storz, Tuttlingen, Germany) or an endotracheal tube or removed with the laryngeal mask together. The roots of the tumors were then cauterized using argon plasma coagulation (ERBE, Tubingen, Germany) or cryotherapy (ERBE, Tubingen, Germany). To avoid airway fire, the concentration of inhaled oxygen was maintained below $40 \%$ during thermal ablation.

Patients were positioned supine and fasted for $6 \mathrm{~h}$ after the interventional bronchoscopic therapy. To ensure clearance of all necrotic tissues, all patients were assessed using bronchoscopy 3-4 days after the procedure.

\section{Assessment and follow-up}

The American Thoracic Society Dyspnea Index (ADI) was used to evaluate the clinical effects of the interventional bronchoscopic therapy. Procedure-related complications were monitored by evaluating any complications that were recorded in the patients' bronchoscopy records, and then evaluating whether these complications were related to the 
Table 1 General information and clinical features of 7 patients with tracheobronchial schwannoma $(n=7)$

\begin{tabular}{|c|c|c|c|c|c|c|c|c|c|}
\hline Case & Gender & Age (years) & \multicolumn{3}{|c|}{ Bronchoscopy findings } & \multicolumn{2}{|c|}{ CT findings } & $\begin{array}{l}\text { LR of } \\
\text { tumour }\end{array}$ & $\begin{array}{c}\text { Follow-up } \\
\text { (years) }\end{array}$ \\
\hline 1 & Female & 59 & Upper trachea & Single & Pedunculated & Intraluminal & 1.5 & No & 7 \\
\hline 2 & Male & 32 & Left MB & Single & Pedunculated & Combined & 2 & Yes & Lost \\
\hline 3 & Male & 57 & Lower trachea & Single & Sessile & Combined & 4 & Yes & 12 \\
\hline 5 & Male & 63 & Upper trachea & Multiple & Sessile & Combined & 2 & No & 3 \\
\hline 6 & Male & 37 & Left MB & Single & Sessile & Combined & 1.5 & Yes & 3 \\
\hline 7 & Female & 26 & Right MB & Single & Sessile & Intraluminal & 2 & No & 2 \\
\hline
\end{tabular}

MB, main-stem bronchus; Max-D, maximum diameter; LR, local reappearance.

interventional bronchoscopic therapy.

Follow-up data were based on bronchoscopy and imaging data. Recurrence of the tumor and any other complications after bronchoscopy were recorded. Telephone calls were made to the patients and/or their relatives to obtain the most recent updates of the patient's condition. The final telephone follow-up was completed in June 2020.

\section{Statistical analysis}

SPSS version 20.0 (IBM Corporation, Armonk, NY, USA) was used for data analysis. Quantitative data are expressed as medians. The paired $t$-test was used to compare changes in $\mathrm{ADI}$ before and after the endoscopic excision procedure; $\mathrm{P}<0.05$, was considered to be statistically significant.

\section{Results}

A total of 7 patients ( 5 men and 2 women; median age, 56 years; age range, 26-63 years) with pathologically diagnosed tracheobronchial schwannoma were enrolled. Five patients were diagnosed with tracheobronchial schwannoma for the first time (cases 1-4 and 7), and two cases were recurrence tracheobronchial schwannoma (cases 5 and 6) who were diagnosed with tracheobronchial schwannoma and were administered initial interventional bronchoscopic therapy in another hospital 23 and 6 years ago, respectively.

Shortness of breath was the chief complaint in all the 7 cases, combined with cough and sputum in 3, fever in 1 , and hemoptysis in 1 . According to the classification of tracheal schwannomas proposed by Kasahara et al. (9) and preoperative chest computed tomography (CT), 2 cases (cases 1 and 7) were classified as intraluminal type (tumor that exists only in the intraluminal space), while 4 cases (cases 2-6) were classified as combined type (tumors that occur in both intraluminal and extraluminal spaces). Bronchoscopic findings revealed smooth, solid, round, or oval enveloped tumors, with maximum diameters approximately $1.5-4 \mathrm{~cm}$. Solitary tumors were seen in six cases, and one case had multiple tumors (case 5, tumors were found in both the upper and middle trachea, and patient was also diagnosed with thyroid schwannoma). The locations of the tumors were as follows: the trachea $(\mathrm{n}=3)$, left main-stem bronchus $(\mathrm{n}=3)$, and right mainstem bronchus ( $\mathrm{n}=1)$.

The general information and clinical features of the 7 patients are summarized in Table 1. The typical manifestations on chest computed tomography and bronchoscopy of tracheobronchial schwannoma cases are shown in Figure 1. The manifestations on bronchoscopy of case 1-7 are shown in Figure 2.

The duration of the operation was between 20 and 280 minutes (median, $137 \mathrm{~min}$ ). Little bleeding occurred during the excision process (approximately $2-10 \mathrm{~mL}$ ). Four patients experienced mild glottic edema during the operation, which was relieved after local spraying with dexamethasone $(5 \mathrm{mg})$. No other procedure-related complications were observed.

After operation, shortness of breath was relieved in all the 7 cases, with the mean ADI score decreasing from $2.29 \pm 0.76$ to $0.29 \pm 0.49(\mathrm{t}=6.481 ; \mathrm{P}=0.001)$. Four patients complained of small amounts of bloody sputum, and 4 complained of sore throat, which were all relieved without special treatment 2-3 days after operation. No serious complications such as asphyxia, airway collapse, or airway 

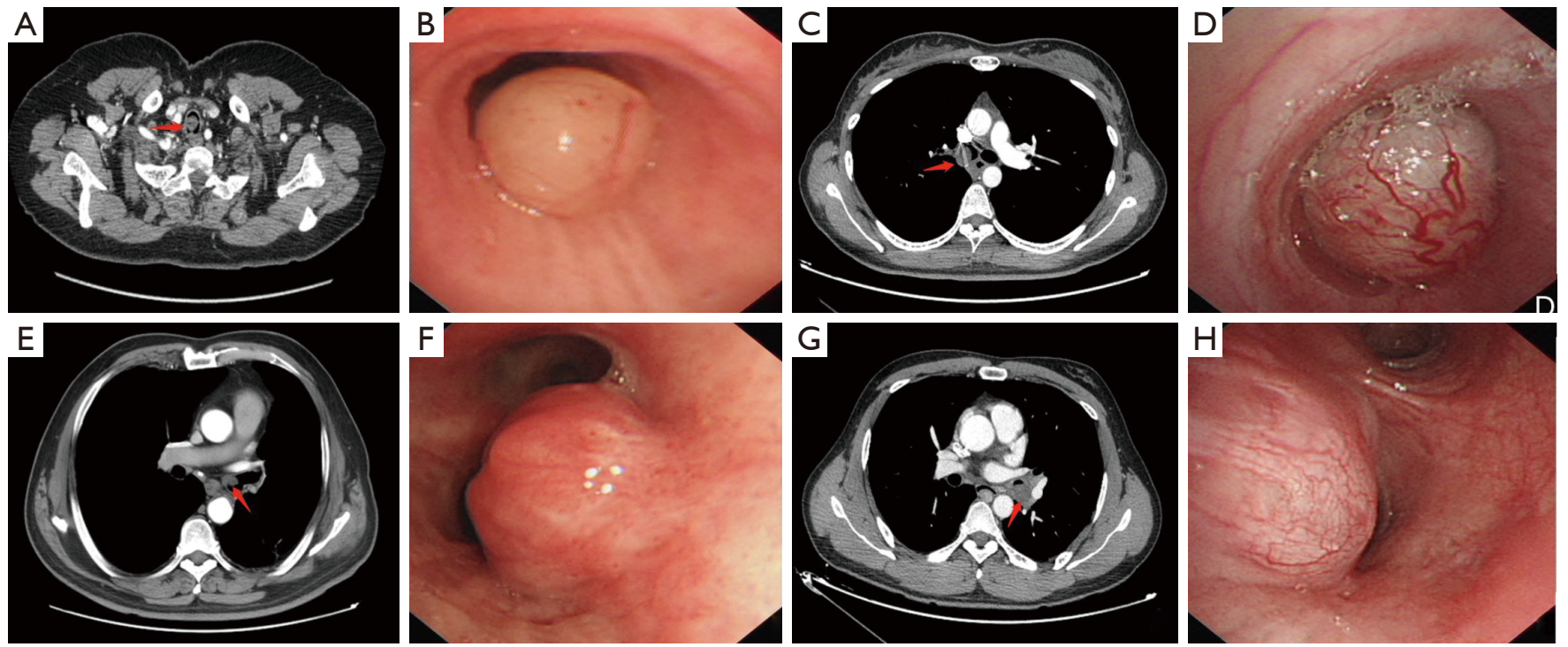

Figure 1 The typical manifestation on chest CT and bronchoscopy of tracheobronchial schwannoma cases (the red arrow marks the location of the tumor on CT scan). (A,B) An intraluminal type pedunculated schwannoma in the upper trachea; (C,D) an intraluminal type sessile schwannoma in the right main-stem bronchus; (E,F) a combined type pedunculated schwannoma in the left main-stem bronchus; (G,H) a combined type sessile schwannoma in the left main-stem bronchus. CT, computed tomography.

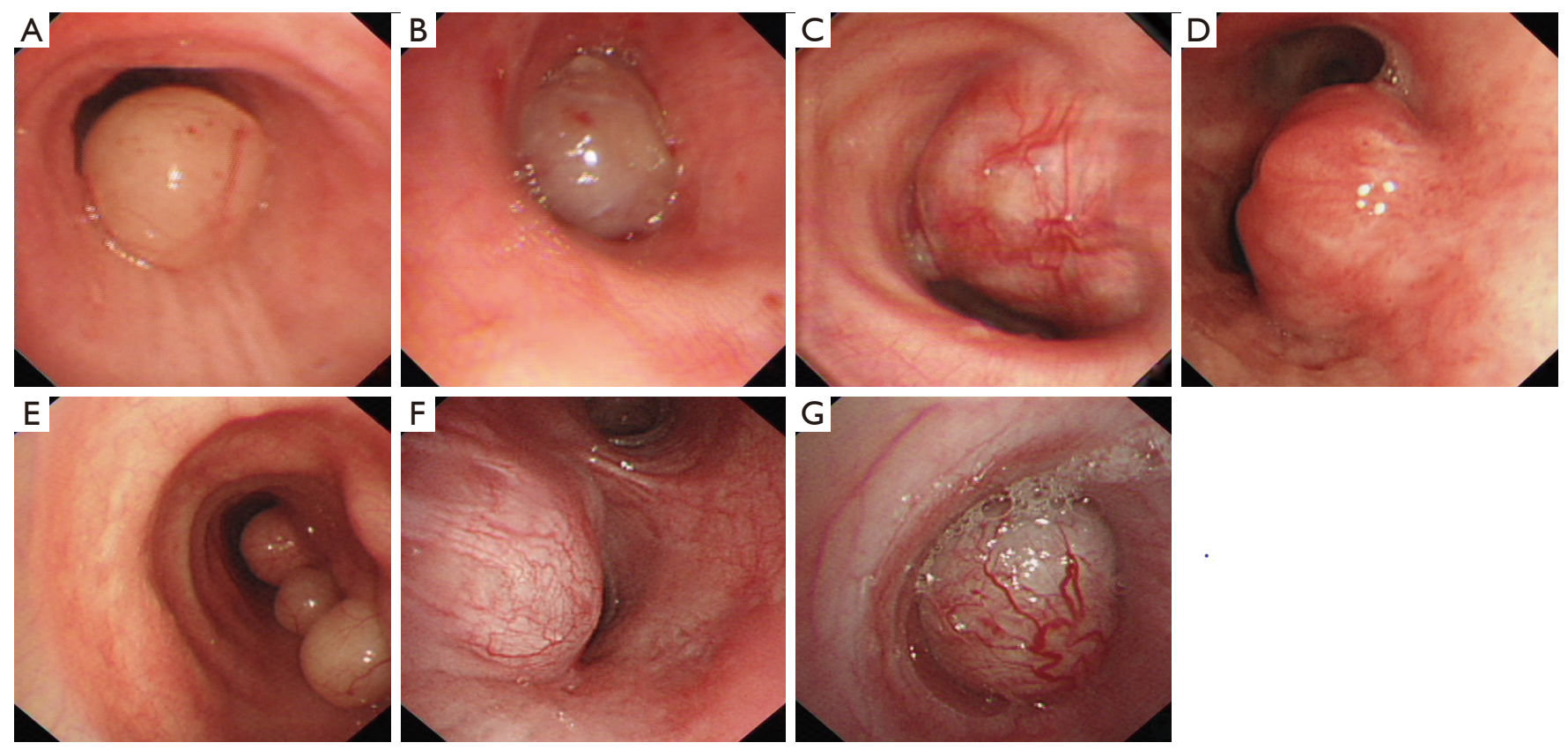

Figure 2 The manifestation on bronchoscopy of cases 1-7 (A,B,C,D,E,F,G, respectively).

perforation occurred.

Intraluminal tumors were found subsequently in 3 cases (cases 2, 3, and 6) during the short-term followup (4 days to 1 month). All the 3 cases had a combined type of schwannoma, and the extraluminal part of the tumor was large. Based on the slow growth characteristics of schwannomas, it was speculated that the intraluminal tumor that reappeared was the extraluminal part that 

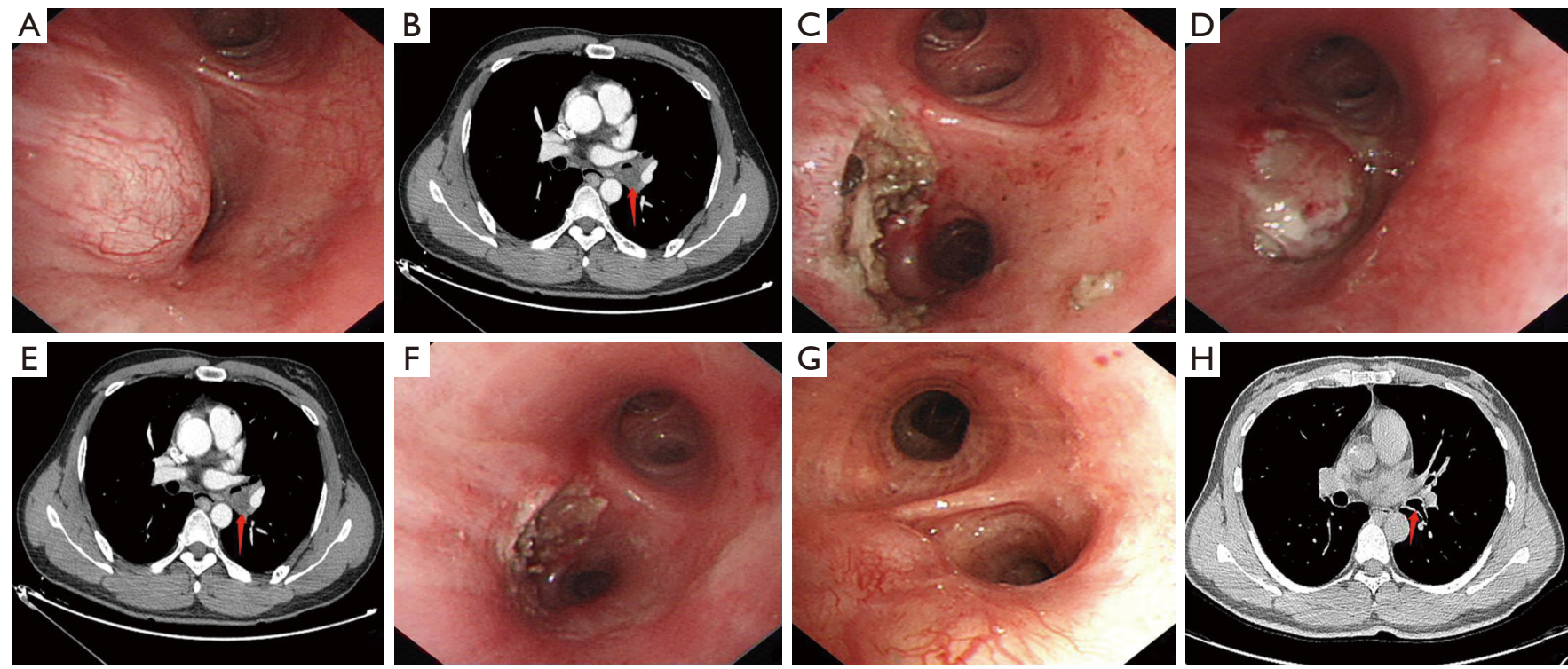

Figure 3 The typical manifestation on chest CT and bronchoscopy of one case (case 6) who received interventional bronchoscopic therapies twice in a short time (the red arrow marks the location of the tumor on CT scan). (A,B) The manifestation on bronchoscopy and chest CT before therapy; (C) the manifestation on bronchoscopy after initial interventional bronchoscopic therapy; (D) follow-up bronchoscopy found intraluminal tumor reappeared 4 days later; (E) CT scan showed the extraluminal part of tumor squeezed into intraluminal space again; (F) the manifestation on bronchoscopy after the second interventional bronchoscopic therapy; $(\mathrm{G}, \mathrm{H})$ the manifestation on bronchoscopy and chest CT 1 month later after the second interventional bronchoscopic therapy. CT, computed tomography.

squeezed into the intraluminal space again after endoscopic resection of intraluminal lesions. Case 2 underwent surgery and the other two cases (cases 3 and 6) received a second interventional bronchoscopy therapy, according to the patients' wishes. No serious complications such as asphyxia, airway collapse, or airway perforation occurred during the second interventional bronchoscopy therapy.

Six patients received long-term follow-up, and one patient was lost to follow-up (case 2). The follow-up period ranged from 2 to 12 years, with a median of 4.5 years. The two patients who received interventional bronchoscopic therapies twice (cases 3 and 6) were followed up for 3 years and 12 years, respectively. As of last contact (December 2020), all patients were in a stable condition without respiratory symptoms.

The typical manifestations on chest computed tomography and bronchoscopy in one case (case 6) who received interventional bronchoscopic therapies twice in a short time are shown in Figure 3.

\section{Discussion}

Tracheobronchial schwannoma is a rare benign airway tumor that accounts for less than $0.5 \%$ of airway tumors $(3,10)$. It originates from Schwann cells of the peripheral nerve sheath and grows slowly. It can occur in all regions of the tracheobronchial tree. When the lesion is located in the trachea or in the proximal bronchus (tracheobronchial schwannoma) and visible by bronchoscopy, the lesion can be classified as the central type (9).

Patients with tracheobronchial schwannoma do not typically experience specific clinical manifestations, which are often misdiagnosed as asthma or bronchitis due to shortness of breath $(11,12)$. The clinical manifestations of intraluminal schwannomas of the trachea depend on the site, size, and extent of obstruction produced by the tumor (13). The most common site for neurogenic tumors is the distal trachea, followed by the proximal and the middle third (5). However, in this study, tumors were located mainly in the main bronchus $(n=4)$, secondary to the proximal trachea $(\mathrm{n}=2)$ and distal trachea $(\mathrm{n}=1)$. This finding was inconsistent with those of literature reports, which may be due to the small size of the sample.

Tracheobronchial schwannoma is characterized by a single solid tumor with an envelope (13); however, Dumoulin $e t a l$. described a case of multiple endobronchial 
schwannomas at three different sites in a single patient (14). In this study, there was also one case of multiple schwannomas in the trachea (case 5). For patients diagnosed with multiple endotracheal or endobronchial schwannomas, a differential diagnosis considering neurofibromatosis is required $(5,15)$. The typical manifestation of tracheobronchial schwannoma on bronchoscopy often exhibits a series of benign tumor imaging findings, such as a smooth surface, clear borders, and no surrounding tissue invasion. However, some patients' tumors exhibit "invasive" imaging features on chest CT, which are characterized by intraluminal and extraluminal growth patterns due to the tumor's origin, and extraluminal progression can lead to recurrence after endoscopic resection of intraluminal lesions (9). This is a special growth pattern of schwannomas and does not represent malignant biological behavior.

As previously mentioned, tracheobronchial schwannomas can be divided into two subtypes: intraluminal and combined. In our study, two patients' preoperative chest CT showed that tumors grew inside the lumen and 5 grew both inside and outside, accounting for $28.6 \%$ and $71.4 \%$ of all cases, respectively. However, findings suggest that about half of the cases of intraluminal schwannomas have non-apparent extraluminal lesions (9). This suggests that the classification according to the CT scan may not always be completely accurate. However, chest CT scan, especially enhanced chest CT scan, is still the most important method to identify tumors, which could also provide abundant information for doctors, including tumor size, location, blood supply, and the relationship between the tumor and surrounding tissues (16). In this study, the chest CT scan could also provide information about the size of extraluminal tumors, which helps doctors to evaluate the possibility of reappearance of intraluminal tumors in a short-term follow-up.

In previous studies, many endoscopic techniques, such as laser with or without carbon dioxide, electronic snaring, APC, cryotherapy, and rigid bronchoscopy have been described to be applied to resect tracheobronchial schwannoma $(7,13,17,18)$. In this study, the main part of the tracheobronchial schwannoma was usually resected using an electrocautery snare (pedunculated schwannoma) or highfrequency electric knife (sessile schwannoma), then APC or cryotherapy can be applied to treat residual tumors. We do not recommend the use of APC or cryotherapy alone or perform mechanical debridement by rigid bronchoscopy to ablate the entire tracheobronchial schwannomas due to their hard texture.
There is no consensus on the optimal management of tracheobronchial schwannomas because of the rarity of this tumor. Although surgical resection is the best option for patients with no reported cases of recurrence and is recommended for patients with sessile tumors and low surgical risk, or extraluminal extension (13), the choice of treatment is still influenced by the risk of tracheal resection. In contrast, compared with surgery, patients who underwent endoscopic resection experienced less trauma and shorter postoperative recovery time. In this study, five cases presented with sessile tumors and six had extraluminal extension, and the follow-up results suggest that for these patients, interventional bronchoscopic therapy may be also a useful and acceptable alternative to surgical resection. Incomplete resection of the lesion may lead to local recurrence. Ge et al. reviewed the literature between 1950 and 2013 and identified 51 cases of primary tracheal schwannoma (13). Nineteen patients underwent endoscopic resection of the mass, of which 4 were reported to have recurrence and were further treated by surgery. It is difficult to determine the recurrence rate because the time of recurrence is very variable, and most of the time, patients were not followed up long enough. Horovitz et al. reported a case of a 38-year-old Caucasian woman who experienced recurrence 12 years after an initial endoscopic excision (19). In our study, there were 2 cases of recurrent tracheobronchial schwannoma (cases 5 and 6). They had received initial endoscopic excision in outside hospitals 23 and 6 years before, respectively, suggesting that patients treated in this manner should be kept under long-range bronchoscopic surveillance. However, surgery may not be the only treatment method for recurrent cases, especially for patients in poor conditions. Our experience emphasizes on the benign nature and slow growth characteristic of tracheal schwannomas illustrating that a second endoscopic resection can still be considered after recurrence.

The major limitations of this study were also recognized. First, it was a single-center retrospective study, making it difficult to determine the generalizability of our findings. Second, due to the rarity of the disease, the sample size was small. Third, the data on recurrence of patients still need to be followed due to the limitation of the follow-up period.

\section{Conclusions}

In conclusion, interventional bronchoscopic therapy is an acceptable approaches for the management of tracheobronchial schwannoma and is a useful alternative to 
surgical resection, especially for those who are at a high risk of surgery or are unwilling to undergo surgery. However, long-range bronchoscopic surveillance is necessary because of the possibility of tumor recurrence. Due to its benign nature, repeat endoscopic resection can still be considered after recurrence.

\section{Acknowledgments}

We thank Min Xu and Chenyang Zhang for their assistance in conducting study and collecting data.

Funding: This study was supported by the Beijing Municipal Administration of Hospitals Incubating Program (grant number: PX2021022).

\section{Footnote}

Reporting Checklist: The authors have completed the STROBE reporting checklist. Available at http://dx.doi. org/10.21037/apm-21-630

Data Sharing Statement: Available at http://dx.doi. org/10.21037/apm-21-630

Peer Review File: Available at http://dx.doi.org/10.21037/ apm-21-630

Conflicts of Interest: All authors have completed the ICMJE uniform disclosure form (available at http://dx.doi. org/10.21037/apm-21-630). Dr. TW reports grants from Beijing Municipal Administration of Hospitals Incubating Program (grant number: PX2021022). The other authors have no conflicts of interest to declare.

Etbical Statement: The authors are accountable for all aspects of the work in ensuring that questions related to the accuracy or integrity of any part of the work are appropriately investigated and resolved. The study was approved by the Institutional Review Board of Beijing Tian Tan Hospital (approval ID: JS2013-007-02). All patients provided written informed consent for the data to be used in this study. The study was conducted in accordance with the Declaration of Helsinki (as revised in 2013).

Open Access Statement: This is an Open Access article distributed in accordance with the Creative Commons Attribution-NonCommercial-NoDerivs 4.0 International License (CC BY-NC-ND 4.0), which permits the non- commercial replication and distribution of the article with the strict proviso that no changes or edits are made and the original work is properly cited (including links to both the formal publication through the relevant DOI and the license). See: https://creativecommons.org/licenses/by-nc-nd/4.0/.

\section{References}

1. Chávez-Fernández DA, Zúñiga-Garza E, LópezSaucedo RA. Primary tracheal schwannoma resected in a Turner syndrome patient: a case report. J Surg Case Rep 2020;2020:rjaa430.

2. Hamdan AL, Moukarbel RV, Tawil A, et al. Tracheal schwannoma: a misleading entity. Middle East J Anaesthesiol 2010;20:611-3.

3. Han DP, Xiang J, Ye ZQ, et al. Primary tracheal schwannoma treated by surgical resection: a case report. J Thorac Dis 2017;9:E249-E252.

4. Melendez J, Cornwell L, Green L, et al. Treatment of large subglottic tracheal schwannoma with microdebrider bronchoscopy. J Thorac Cardiovasc Surg 2012;144:510-2.

5. Rusch VW, Schmidt RA. Tracheal schwannoma: management by endoscopic laser resection. Thorax 1994;49:85-6.

6. Le Rouzic O, Ramon PP, Bouchindhomme B, et al. Benign trachobronchial schwannoma treated by complete endoscopic resection followed by cryotherapy. Rev Mal Respir 2011;28:88-91.

7. Isaac BT, Christopher DJ, Thangakunam B, et al. Tracheal schwannoma: Completely resected with therapeutic bronchoscopic techniques. Lung India 2015;32:271-3.

8. Hamouri S, Novotny NM. Primary tracheal schwannoma a review of a rare entity: current understanding of management and followup. J Cardiothorac Surg 2017;12:105.

9. Kasahara K, Fukuoka K, Konishi M, et al. Two cases of endobronchial neurilemmoma and review of the literature in Japan. Intern Med 2003;42:1215-8.

10. Grillo HC, Mathisen DJ. Primary tracheal tumors: treatment and results. Ann Thorac Surg 1990;49:69-77.

11. Thomas R, Christopher DJ, Thangakunam B, et al. Tracheal schwannoma as a mimic of bronchial asthma. Singapore Med J 2012;53:e95-96.

12. Oliveira RC, Nogueira T, Sousa V, et al. Bronchial schwannoma: a singular lesion as a cause of obstructive pneumonia. BMJ Case Rep 2016;2016:bcr2016217300.

13. Ge X, Han F, Guan W, et al. Optimal treatment for primary benign intratracheal schwannoma: A case report 
and review of the literature. Oncol Lett 2015;10:2273-6.

14. Dumoulin E, Gui X, Stather DR, et al. Endobronchial schwannoma. J Bronchology Interv Pulmonol 2012;19:75-7.

15. Joshi R. Learning from eponyms: Jose Verocay and Verocay bodies, Antoni A and B areas, Nils Antoni and Schwannomas. Indian Dermatol Online J 2012;3:215-9.

16. Parsons RB, Milestone BN, Adler LP. Radiographic assessment of airway tumors. Chest Surg Clin N Am 2003;13:63-77, v-vi.

Cite this article as: Jin B, Wang T, Wang J, Qiu X, Pei Y, Wang Y, Zhang J. Interventional bronchoscopic therapy in adult patients with tracheobronchial schwannoma. Ann Palliat Med 2021;10(6):6279-6286. doi: 10.21037/apm-21-630
17. Lee BR, Choi YD, Kim YI, et al. Endobronchial schwannoma treated by rigid bronchoscopy with argon plasma coagulation. Tuberc Respir Dis (Seoul) 2012;73:174-7.

18. Jung YY, Hong ME, Han J, et al. Bronchial schwannomas: clinicopathologic analysis of 7 cases. Korean J Pathol 2013;47:326-31.

19. Horovitz AG, Khalil KG, Verani RR, et al Primary intratracheal neurilemoma. J Thorac Cardiovasc Surg 1983;85:313-7. 